\title{
Qualification of Customised Medical Implants Produced by Ti6AI4V(ELI) Additive Manufacturing
}

\author{
W B du Preez ${ }^{a},{ }^{*}$ and G J Booysen ${ }^{\mathrm{b}}$ \\ ${ }^{\mathrm{a}}$ Department of Mechanical and Mechatronics Engineering \\ ${ }^{b}$ Centre for Rapid Prototyping and Manufacturing \\ Central University of Technology, Free State \\ Bloemfontein, South Africa \\ wdupreez@cut.ac.za
}

\begin{abstract}
Although many cases of medical implants produced through additive manufacturing (AM) in Ti6Al4V have been reported in literature, most of these processes had not been qualified. To enable certification and commercialisation of medical implants and devices an ISO 13485:2016 quality management system was successfully implemented in the Centre for Rapid Prototyping and Manufacturing (CRPM) at the Central University of Technology, Free State in South Africa. This certification covers qualification of both design, development and production of patient specific custom made titanium implants, as well as preoperative models, jigs and cutting guides in nylon by means of AM and supports commercialisation.
\end{abstract}

With this quality management system as framework for ensuring the reliability and repeatability of the AM performed at the CRPM, the generation of data to validate the individual processes in the AM process chain was pursued. Sufficient research data has been produced and published to prove that medical implants produced through AM can fully comply with the international standards for material, physical, chemical and mechanical properties. In this paper the research performed towards the qualification of AM of Ti6Al4V medical implants is discussed. Examples are given of internationally leading work on utilising these implants in maxillofacial and orthopaedic surgeries.

\section{Introduction}

Over the past decade the Centre for Rapid Prototyping and Manufacturing (CRPM) of the Central University of Technology, Free State has established itself as the leading South African player in the production of customised Ti6AI4V (ELI) medical implants produced through additive manufacturing. In collaboration with leading maxillofacial and orthopaedic surgeons the engineers of the CRPM have designed and produced through direct metal laser sintering (DMLS) of Ti6AI4V (ELI) powder, numerous implants that have been successfully utilised in facial and skeletal reconstruction [1]. However, it was realised at an early stage that full acceptance of this disruptive technology by industry would require quality management across the product life cycle and dedicated research to prove the reliability and repeatability of the DMLS production process chain. Such a drive towards full qualification of medical implants produced through DMLS would align strongly with the priorities identified in the South African Additive Manufacturing Strategy [2].

\section{Establishment of an ISO 13485 Compliant Quality Management System}

As mentioned above, in offering AM as an implant manufacturing solution, the complete process chain (design, manufacturing and post processing) had to be investigated to develop a certified manufacturing solution. Three objectives were set to address this complete process certification. A process chain was introduced as part of the research methodology as shown in Figure 1. 


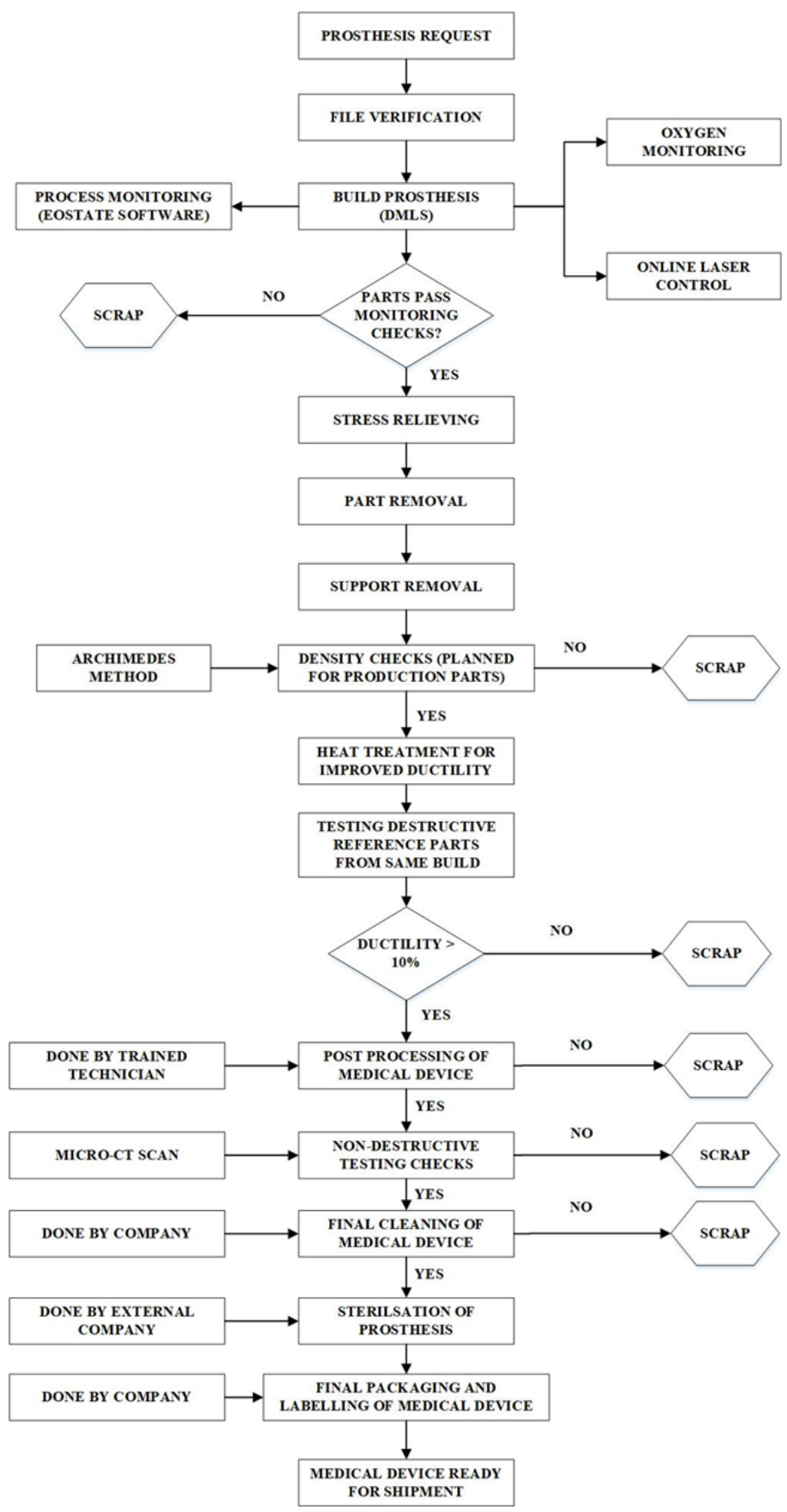

Figure 1: The CRPM certification process chain 
Objective 1 addressed the risk identification and ways to mitigate these risks through developing procedures, standard operating procedures (SOPs) and supporting documents. This can be seen as the technical certification of this certified manufacturing solution. In the medical device industry risk management is critical throughout the entire life cycle of a device and it is vital to successfully implement a risk management system to ensure that a safe, effective product is manufactured on time and within budget. ISO 14971:2012 [3] is the harmonised standard for risk management which defines the international requirements of risk management systems for medical devices. Using ISO 14971:2012 and ISO 13485:2016 [4] as guidelines, it is important to develop a Risk Management Procedure, including relevant flow diagrams, to define how the AM producer will do risk assessment and management. The complexity of the process can be seen in Figure 2.

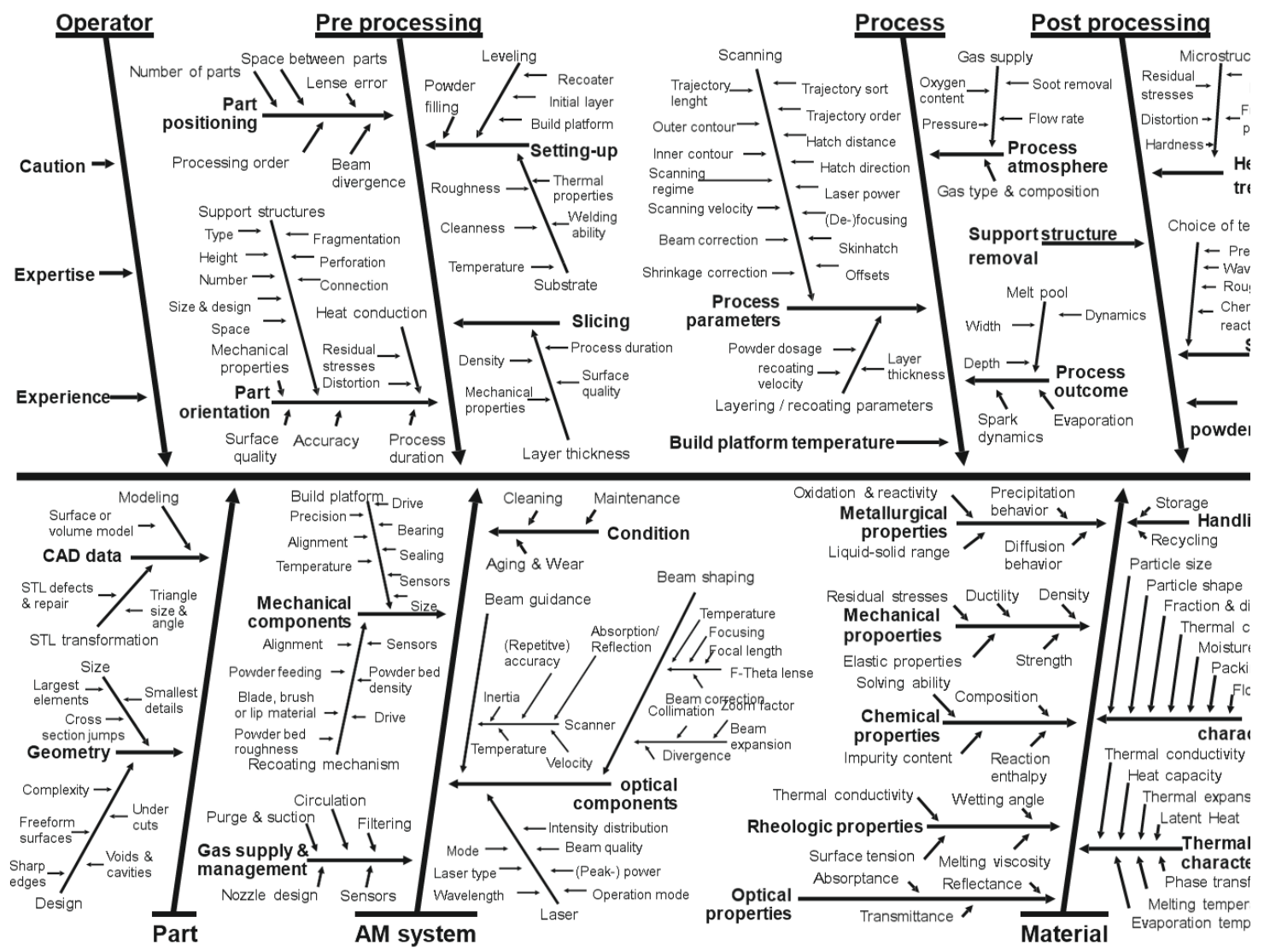

Figure 2: Factors which influence the DMLS process. Courtesy of Dr. Christoph Haberland, Siemens

Objective 2 focussed on developing an integrated documentation framework, keeping traceability and repeatability in mind. Process and machine validation requirements were developed that form part of the quality certification process and are used to evaluate the consistency of the technical certification to prove repeatability and traceability of the products manufactured.

Objective 3 focussed on identifying shortcomings in the framework and an in-depth analysis on ways to rectify these problems though continual improvement. Throughout this process it was important not only to address some areas of concern, but to explain the methodology behind risk mitigation, procedure and SOP development and validation and how these individual areas link to each other. The ISO $13485: 2016$ system is based on continual improvement principles which would mean that where new risks arise, the process of addressing these risks will be fast-tracked through this framework development.

\section{Research on Additively Manufactured Ti6Al4V (ELI)}


Although this quality management system provides the framework for ensuring the reliability and repeatability of the AM performed at the CRPM, the generation of data to validate the individual processes in the AM process chain is imperative. Sufficient research data had to be produced and published to prove that parts produced through AM could fully comply with the accepted international standards for material, physical, chemical and mechanical properties of such parts. For achieving this, a process chain for qualification of medical implants was developed as shown in Figure 3 . This diagram indicates the different areas (yellow blocks) where the research projects were focused to generate the data and establish the techniques and processes that would underpin the qualification procedures [1].

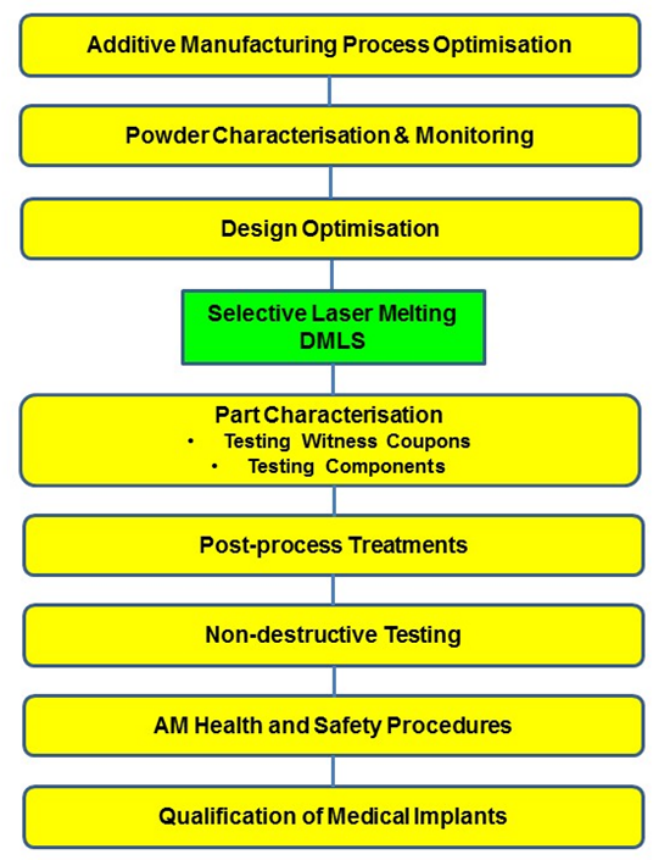

Figure 3: Research areas in the DMLS process chain for qualification of medical implants

\section{DMLS process optimisation}

Based on initial studies [5] and subsequent verification, it was decided not to deviate from the process parameters provided by the supplier, EOS, for the EOSINT M280 (200W) DMLS machine that is dedicated to medical implant production. This parameter set is known to produce samples with high density and microCT-determined microporosity that is evenly distributed and at levels below $0.01 \%[6]$.

Powder characterisation and monitoring

The Ti6Al4V (ELI) powder was characterised as received from the supplier (virgin powder) and the properties were monitored after numerous DMLS build cycles with re-used powder [7]. Table 1 summarises the powder characterisation methods used in this study, which all complied with the international standards specified in the ASTM F3049-14 standard guide for characterising properties of metal powders used for additive manufacturing processes.

Table 1: Techniques and methods used for powder characterisation

\begin{tabular}{|l|l|l|}
\hline \multicolumn{1}{|c|}{ Characteristic } & \multicolumn{1}{|c|}{ Method/Technique } & Instrument \\
\hline Elemental composition & $\begin{array}{l}\text { Inductively coupled plasma-optical emission } \\
\text { spectroscopy (ICP-OES) }\end{array}$ & SPECTRO ARCOS \\
\hline Gas content & Inert gas fusion & ELTRA OHN 2000
\end{tabular}




\begin{tabular}{|l|l|l|} 
& LECO combustion & LECO OHN 836 \\
\hline \multirow{2}{*}{ Morphology } & Scanning electron microscopy (SEM) & JEOL JSM-6510 \\
\hline \multirow{2}{*}{ Density } & Laser scattering & MICROTRAC SI/S3500 \\
\cline { 2 - 4 } & microCT scanning & General Electric Nanotom S \\
\cline { 2 - 4 } & Pycnometer & AccuPyc II 1340 \\
\cline { 2 - 4 } Cohesion & microCT scanning & General Electric Nanotom S \\
\hline Flowability & Rheology & FT4 powder rheometer \\
\hline
\end{tabular}

DMLS part characterisation

The microstructure, physical and mechanical properties of witness coupons produced through DMLS were determined as-built and after postprocess treatments $[6,8]$ and were found to compare very well with standard properties of wrought material. Tensile test specimens were machined from DMLS bars and tested to ASTM E8/E8M-13a. The v-notches of impact toughness specimens were machined from DMLS test blocks and tested to ASTM E23.

Fatigue test specimens in the as-built state and after heat treatment were produced by laser powder bed fusion in the form of bars with square cross sections and their longest axes in the $\mathrm{X}, \mathrm{Y}$ and $\mathrm{Z}$ orientations, with the Z-direction being the DMLS build direction. Standard process parameters for the EOS M280 system were used with Ti6Al4V (ELI) powder as reported in [9]. A residual stress relieving heat treatment at $650^{\circ} \mathrm{C}$ for $3 \mathrm{~h}$, followed by a high temperature anneal at $950^{\circ} \mathrm{C}$ for $2 \mathrm{~h}$, was used. Fatigue test specimens were machined from the bars according to ASTM E466-15 and subsequently polished in the longitudinal direction on a Morrison Specimen Polishing Machine using an 800 grit paper for 15 minutes, followed by a 1200 grit paper for 20 minutes, resulting in samples with a smooth finish [9].

The Split Hopkinson Pressure Bar test was used to study the high strain rate properties of as-built and stress relieved DMLS Ti6Al4V (ELI) in both tension and compression $[10,11]$.

\section{$\underline{\text { Results }}$}

Quality management results for Objective 1

In this research project, a total of 68 risks were identified in the following areas: design, machine setup, powder handling, SLM process, part removal, density checks, heat treatment, non-destructive testing, destructive testing, surface finishing and coating, cleaning, sterilisation and packaging. The action plan was to mitigate these risks by developing procedures, SOPs, supporting documents and where needed, full machine and process validation.

\section{Quality management results for Objective 2}

Nineteen procedures, 34 SOPs, 5 supportive protocols, 3 machine validations and 5 process validations were identified and developed. The risks were plotted against possible procedure, SOP's, document developments and validation protocols. An example is shown in Table 2 on how these risks could be mitigated. It can be seen in this example that some risks, pertaining to the design process, could be mitigated by a combination of developing a procedure, SOP, protocol, medical device file, design rules and full process or software validation.

Table 2: Example of design risk mitigation 


\begin{tabular}{|c|c|c|c|c|c|c|c|c|c|}
\hline \multicolumn{10}{|c|}{ Design } \\
\hline \multirow{2}{*}{\multicolumn{2}{|c|}{$\begin{array}{l}\begin{array}{l}\text { Risk } \\
\text { Classification }\end{array} \\
\text { Risk } \\
\text { Description }\end{array}$}} & \multirow[t]{2}{*}{$\begin{array}{l}\text { Internal - INT } \\
\text { External = EXT }\end{array}$} & \multicolumn{4}{|c|}{ INT } & \multicolumn{3}{|c|}{ EXT } \\
\hline & & & $\begin{array}{l}\text { Flipped } \\
\text { Import }\end{array}$ & $\begin{array}{l}\text { Slice Data } \\
\text { to STL }\end{array}$ & $\begin{array}{l}\text { Over- } \\
\text { or }\end{array}$ & $\begin{array}{l}\text { Design } \\
\text { not }\end{array}$ & $\begin{array}{l}\text { Wrong } \\
\text { Patient }\end{array}$ & $\begin{array}{c}\text { Bad } \\
\text { Quality }\end{array}$ & $\begin{array}{l}\text { Incorrect } \\
\text { Doctor's }\end{array}$ \\
\hline \multirow{8}{*}{ 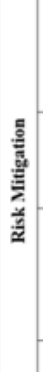 } & \multirow[b]{2}{*}{ Procedures } & \multirow{2}{*}{$\begin{array}{l}\text { Product } \\
\text { Development }\end{array}$} & $\mathrm{x}$ & & $\mathrm{x}$ & & & & \\
\hline & & & & $\mathrm{X}$ & & & & & \\
\hline & \multirow{2}{*}{$\begin{array}{l}\text { Standard } \\
\text { Operating } \\
\text { Procedures }\end{array}$} & $\begin{array}{l}\text { Process } \\
\text { Overview }\end{array}$ & & & $\mathrm{x}$ & $\mathrm{X}$ & & $\mathrm{X}$ & \\
\hline & & Implant Design & $\mathrm{x}$ & & & & & & \\
\hline & \multirow{3}{*}{$\begin{array}{l}\text { Develop } \\
\text { Documents }\end{array}$} & $\begin{array}{l}\text { Patient CT } \\
\text { Scan Protocol }\end{array}$ & & & & & & $\mathrm{X}$ & \\
\hline & & $\begin{array}{l}\text { Medical Device } \\
\text { File }\end{array}$ & & & $\mathrm{x}$ & & $\mathrm{x}$ & & $\mathrm{x}$ \\
\hline & & Design Rules & & & & $\mathrm{x}$ & & & \\
\hline & Validation & Software & & $\mathrm{x}$ & & & & & \\
\hline
\end{tabular}

\section{Quality management results for Objective 3}

Process risk assessments were done before and after the framework development and this showed a significant reduction in the risk index. The risk index is the level of risk multiplied by the probability of occurrence. Because these patient-specific implants are manufactured in relatively low volumes, the probability of occurrence numbers will be significantly lower than in a mass production environment.

Research based validation

From the systematic characterization of virgin Ti6AI4V (ELI) powder and monitoring of its properties after re-use $[7,12,13]$ it was concluded that, provided the quality management system is maintained, the un-used Ti6AI4V (ELI) powder in the DMLS system can be re-used until fully consumed. This has positive implications for the economics of producing customised medical implants through DMLS.

Charpy impact tests showed the v-notch toughness of DMLS Ti6Al4V (ELI) at ambient temperature to increase by about $21 \%$ upon stress relieving heat treatment. However, this is still $22 \%$ lower than the value recommended for use in the aerospace industry. This indicates a need for further post-processing of DMLS parts for application in higher specification industries, such as the aerospace industry. The values of v-notch toughness at the temperature of $-130^{\circ} \mathrm{C}$ was about $70 \%$ and $59 \%$ of the values obtained at room temperature for as-built and stress relieved samples, respectively. This clearly indicates that the alloy still retains appreciable toughness even at sub-zero temperatures $[10,14]$.

A stress relieving heat treatment of 3 hours at $650^{\circ} \mathrm{C}$ followed by a high temperature annealing heat treatment at $950^{\circ} \mathrm{C}$ for $2 \mathrm{~h}$ was found to produce a bimodal microstructure, considered to give optimal fatigue properties [9]. The high cycle fatigue crack initiation of these heat treated DMLS Ti6Al4V (ELI) specimens started from surface and sub-surface DMLS process-related pores. The fatigue life of these specimens mainly depended on the area of the process-related crack initiation pores projected on the fracture plane. Pores with large crack initiation areas had lower numbers of cycles to failure than those with small crack initiation areas loaded at the same stress loads [9]. Critical pores for cyclic applications in metal additive manufacturing are those near the surface, specifically quantified to be within $0.06 \mathrm{~mm}$ from the surface. Direct correlation of fatigue crack initiating pores in microCT data allowed detailed analysis of characteristics of the 'killer' pores. It was found that size and shape are less important than surface proximity.

The Split Hopkinson Pressure Bar results showed the flow stress of Ti6Al4V (ELI) to increase at higher strain rate loading, while the fracture strain decreased, for both as-built and stress relieved specimens (Figure 4). Nevertheless, the relative strain rate sensitivity of the stress-relieved specimens was higher than that of as-built specimens [10,11]. Failure analysis of the deformed surfaces indicated that the two forms of the alloy failed as a result of the development of adiabatic shear bands when exposed to compressive high strain rate loading [10,15]. Tensile fracture of the two forms of the alloy was found to be related to surface and internal defects and pores formed during the DMLS process. These acted as crack initiation and propagation sites. 

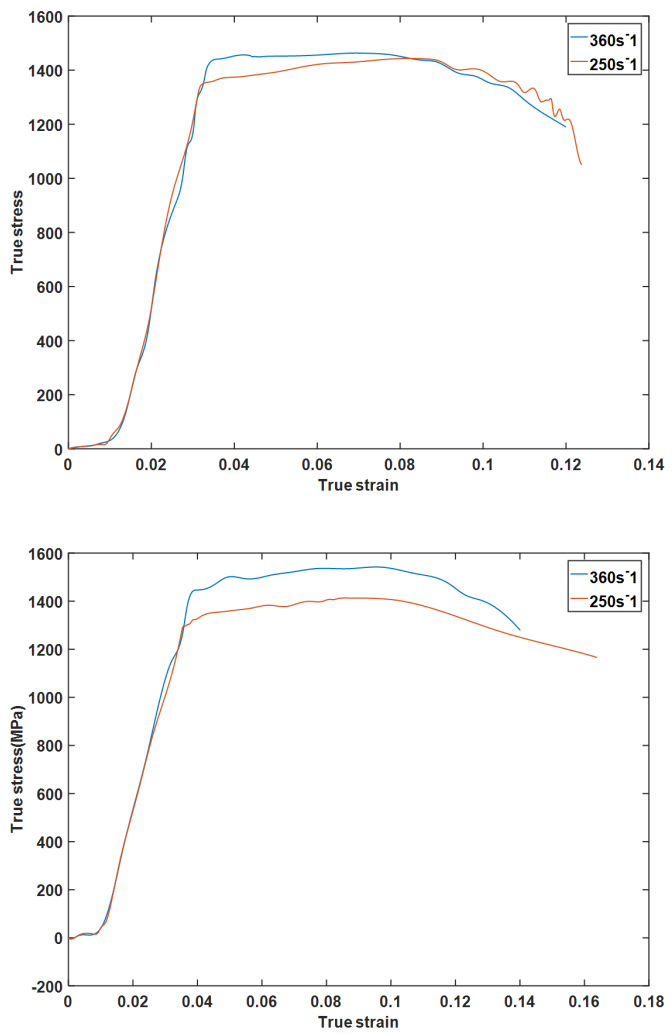

(a)

(b)

Figure 4: The true stress-strain for (a) as-built and (b) stress relieved samples at two different tensile high strain rates

\section{Discussion}

The CRPM was successful in migrating to the revised ISO 13485 (2016 version) [16] and the external audit was completed by TÜV SÜD on 23 March 2018. The International Organisation for Standardisation (ISO) revised the 2012 version, and the ISO 13485:2016 was designed to respond to latest quality management system practices, including changes in technology and regulatory requirements and expectations. TÜV SÜD also audited all the Patient Files, because the CRPM assisted more than 25 patients in the previous two years. The validation data generated through the research of the CRPM since 2015 provided convincing evidence of the reliability and reproducibility of the DMLS process and the post DMLS treatment processes employed in the process chain for producing customised medical implants. Further supportive results for the qualification of the process chain was recently published [17]. This resulted in a positive outcome of the audit, confirming that CRPM can deliver certified Ti6Al4V (ELI) medical implants based on a qualified production process chain. Some of these implants designed and manufactured by CRPM are shown in Table 3.

Table 3: Customised implants manufactured by CRPM through DMLS 


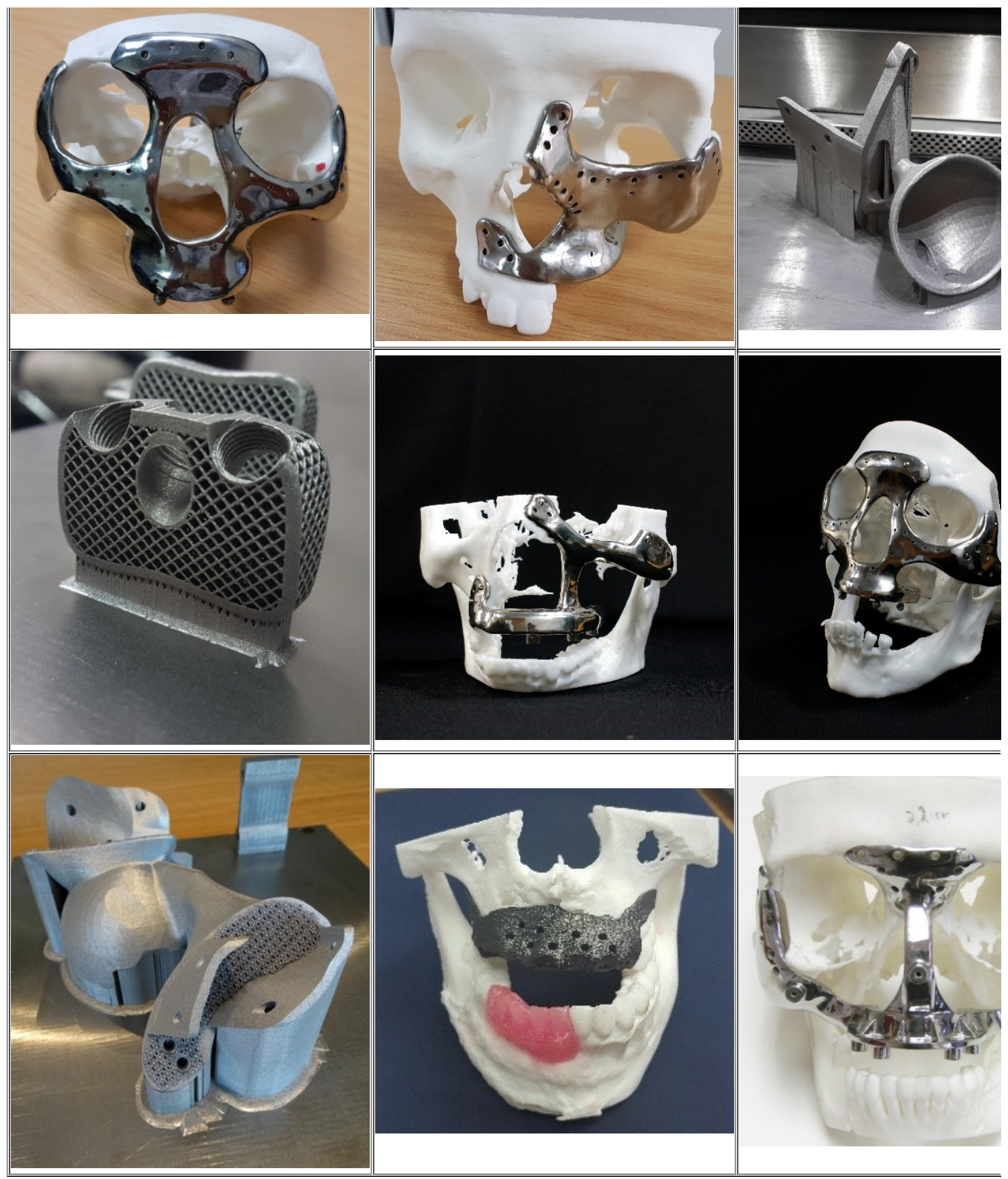


Handling of the feedstock Ti6Al4V (ELI) powder within the ISO 13485:2016 quality management system has led to improved costeffectiveness of the DMLS production process through minimising material wastage and complete consumption of the powder. Through DMLS process control and control of qualified post-DMLS processes, a qualified process chain, supported by validation data generated through research on processes and products, for production of customised medical implants under the ISO 13485:2016 quality management system of the CRPM was established. On-going research is aimed at further improving the in-depth insight in the performance of the DMLS produced parts under more severe dynamic operational conditions, such as those experienced by structural aerospace components.

\section{Acknowledgements}

This research was supported and funded by the South African Department of Science and Technology through the Collaborative Program in Additive Manufacturing, Contract No.: CSIR-NLC-CPAM-15-MOA-CUT-01.

The support of the South African Research Chairs Initiative of the Department of Science and Technology and National Research Foundation of South Africa (Grant No.: 97994) is acknowledged.

The financial support of the Carl and Emily Fuchs Foundation for the reconstructive surgery cases is gratefully acknowledged.

\section{$\underline{\text { References }}$}

[1] W B du Preez, JOM, 71(2) (2019) 655 - 661

[2] D. de Beer, W. du Preez, H. Greyling, F. Prinsloo, F. Sciammarella, N. Trollip, M. Vermeulen, with contributions from Terry Wohlers, "A South African Additive Manufacturing Strategy", Department of Science and Technology, April 2016, http://www.rapdasa.org/wpcontent/uploads/2017/02/South-African-Additive-Manufacturing-Strategy. Accessed 3 April 2019.

[3] International Standards Organisation. 2012. 14971: Medical devices - Application of risk management to medical devices. Switzerland: International Standards Organisation.

[4] International Standards Organisation. 2016. 13485: Medical devices - Quality management systems - requirements for regulatory purposes. Switzerland: International Standards Organisation.

[5] J. Els, Optimal process parameters for Direct Metal Laser Sintering of Ti64 for medical implant production, MTech Dissertation, Central University of Technology, Free State (2016)

[6] P. Krakhmalev, G. Fredriksson, I. Yadroitsava, N. Kazantseva, A. Du Plessis, I. Yadroitsev, Phys. Procedia, 83 (2016) 778-788

[7] K. Thejane, Characterisation and monitoring of Ti6AI4V ELI powder used for the qualification of medical implants produced through additive manufacturing, M.Eng. dissertation, Central University of Technology, Free State (2018)

[8] Moletsane, M.G., Krakhmalev P., Kazantseva, N., Du Plessis, A., Yadroitsava I., Yadroitsev I., S. Afr. J. Ind. Eng. $27(3)$ (2016) 110 - 121

[9] L.B. Malefane, W.B. du Preez, M. Maringa \& A. du Plessis, S. Afr. J. Ind. Eng. 29(3) (2018) $299-311$.

[10] A M Muiruri, Investigation of high strain rate behaviour and Impact toughness of Ti6Al4V (ELI) parts built by the EOS M280 DMLS system with standard process parameters; as built and stress relieved, MEng Dissertation, Central University of Technology, Free State (2019)

[11] AM Muiruri, M Maringa, W B du Preez \& LM Masu, International Conference on Competitive Manufacturing (COMA '19) Proceedings, 30 January to 1 February 2019, Stellenbosch, South Africa, 174-180, ISBN: 978-0-7972-1779-9

[12] K. Thejane, S. Chikosha, W. B. du Preez, S. Afr. J. Ind. Eng. 28(3) (2017) 161-171

[13] A. du Plessis, P. Sperling, A. Beerlink, W.B. du Preez, S.G. le Roux, MethodsX 5 (2018) 1336 - 1345,

[14] A.M. Muiruri, M. Maringa, W.B. du Preez \& L.M. Masu, S. Afr. J. Ind. Eng. 29(3) (2018) $284-298$.

[15] AM Muiruri, M Maringa, WB du Preez, L Masu, Additive Manufacturing of Titanium Parts pre-conference seminar, International Convention Centre, Durban, 7 November 2017, RAPDASA 2017 Conference Proceedings, 165 - 179, ISBN: 978-0-620-77329-4 
[16] CRPM ISO 13485:2016 Quality Management System (in-house document), Central University of Technology, Free State (2018)

[17] I. Yadroitsev, P. Krakhmalev, I. Yadroitsava and A. du Plessis, Qualification of Ti6Al4V ELI alloy produced by laser powder bed fusion for biomedical applications, JOM, 70(3) (2018) 372-377 